\title{
Surgical outcomes of isolated tricuspid valve procedures: repair versus replacement
}

\author{
Julius I. Ejiofor, Robert C. Neely, Maroun Yammine, Siobhan McGurk, Tsuyoshi Kaneko, Marzia \\ Leacche, Lawrence H. Cohn, Prem S. Shekar \\ Division of Cardiac Surgery, Brigham and Women's Hospital, Harvard Medical School, Boston, MA, USA \\ Correspondence to: Prem S. Shekar, MD. Chief, Division of Cardiac Surgery, Brigham and Women's Hospital, 75 Francis St, Boston, MA 02115, USA. \\ Email: pshekar@partners.org.
}

\begin{abstract}
Background: Isolated tricuspid valve (ITV) operations are infrequent and the decision to operate is controversial. We report a series of ITV operations to outline the current disease status requiring this uncommon procedure with an emphasis on the results of tricuspid valve repair (TVr) versus replacement (TVR). Methods: Using our prospective cardiac surgery database, 57 patients who underwent ITV operations between 01/02-03/14 were identified. Median follow up time was 3.5 years [interquartile range (IQR), $0.8-6.7$ years].
\end{abstract}

Results: Fifty-seven patients underwent ITV surgery with a mean age of $54.4 \pm 14.9$ yrs and $61 \%$ were women. Baseline characteristics were similar between patients who underwent TVr $(n=18)$ or TVR (n=39). The etiologies of TV dysfunction were: ITV endocarditis 14/57 (25\%), persistent TV regurgitation after left-sided valve surgery in 12/57 (21\%), traumatic biopsies and iatrogenic injury from pacing leads in $11 / 57$ (19\%), orthotopic heart transplant 9/57 (16\%), carcinoid syndrome 3/57 (5\%), congenital 2/57 (5\%) and idiopathic 5/57 (9\%). Overall, 32/57 (56\%) patients had prior heart surgery; of which 10/32 (31\%) were TV procedures. Bioprosthetic prostheses were used in 34/39 (87\%) patients. Of those who had repair, 11/18 (61\%) had ring annuloplasty, 3/18 (17\%) bicuspidization, and 3/18 (17\%) De Vega annuloplasty and one had vegetectomy. Operative mortality was $5.1 \%(n=2)$ and $16.7 \%(n=3)$ for TVR and TVr groups, respectively $(\mathrm{P}=0.32)$, with an overall mortality rate of $8.6 \%$. Postoperative complications included new onset renal failure in $6 / 39(15 \%)$ of TVr and 2/18 (11\%) of TVR $(\mathrm{P}=0.71)$ and there were no strokes. Overall survival rates and degree of residual $\mathrm{RV}$ dysfunction were similar for the two groups (both $\mathrm{P}=0.3$ ). Five-year survival was $77 \%$ and $84 \%$ for $\mathrm{TVr}$ and TVR respectively $(\mathrm{P}=0.52)$. There was no difference in rates of recurrent tricuspid regurgitation for TVr and TVR (35.7\% vs. $23.5 \%$, respectively, $\mathrm{P}=0.4)$.

Conclusions: ITV surgery is associated with improved but still relatively high operative mortality. Midterm outcomes for TVr and TVR are similar with regards to postoperative complications, survival, and freedom from recurrent tricuspid regurgitation.

Keywords: Tricuspid valve (TV); isolated; endocarditis; tricuspid valve repair (TVr); tricuspid valve replacement (TVR)

Submitted Feb 26, 2017. Accepted for publication Apr 18, 2017.

doi: $10.21037 /$ acs.2017.05.02

View this article at: http://dx.doi.org/10.21037/acs.2017.05.02

\section{Introduction}

In a recent review of the Society of Thoracic Surgeons (STS) database, $86 \%$ of tricuspid valve (TV) operations were performed in conjunction with another major procedure, usually left sided valve operations, highlighting the relative rarity of isolated TV surgery (1). Indications for TV surgery have largely been placed within the context of concomitant left sided heart disease and the presence of pulmonary hypertension (PHTN). The updated American 
Heart Association/American College of Cardiology 2014 guidelines for valvular heart disease recommend isolated tricuspid valve (ITV) repair for severe TR when symptomatic (Class I) or accompanied by progressive right ventricular dysfunction (Class IIb) (2). The guideline also recommend TV repair for asymptomatic severe TR or dilated tricuspid annulus when performing surgery on leftsided valves (2).

In the absence of left sided pathology, the decision to perform an ITV operation is more controversial. The literature on isolated TV operations is sparse and historically associated with high mortality rates $(3,4)$. This is especially true in the setting of right ventricular failure (4). While some studies have questioned if TV replacement is ever indicated (5), there is increasing interest in TV pathology and interventions reflected in recent publications $(1,6-8)$. We present our mid and long term outcomes with ITV surgery to help elucidate the indications, timing, and surgical approach for ITV repair and/or replacement.

\section{Methods}

\section{Patient selection}

All adult patients aged over 17 years who underwent ITV surgery from January 2002 through to March 2014 at Brigham and Women's Hospital were identified from our prospective cardiac surgery database. This study was approved by the Partners Healthcare Institutional Review Board. A total of 57 patients underwent ITV surgery. At our institution, we believe that careful patient selection and preoperative optimization is paramount to excellent results. This includes patients with symptomatic, severe TV regurgitation or asymptomatic with progressive $\mathrm{RV}$ annular dilation $(>40 \mathrm{~mm})$ and/or systolic dysfunction.

Patients' demographics, perioperative characteristics, laboratory test results, and in-hospital outcomes of the index admission were extracted from the Brigham cardiac surgery database and the linked Partners Health Care Electronic System. Variables were defined and coded according to the Society for Thoracic Surgeons, Adult Cardiac Surgery database specifications, version 2.52. Follow up data was aggregated from the linked Partners Health Care System, and primary care physicians or cardiologists documentation. Of the 52 patients alive at discharge, follow up was $92 \%$ complete. Mortality data was obtained from our internal research data repository, our state's Department of Public Health and Registry of Vital Statistics, through routine patient follow-up, or by query of the Social Security Death Index. Median follow-up duration was 3.5 years [interquartile range (IQR), 0.8-6.7 years].

\section{Statistical analysis}

Categorical variables are presented as percentage (number) and were evaluated using Fisher's Exact test. Continuous variables are presented as mean \pm standard deviation if normally distributed or median and IQR if non-normally distributed. Analyses of continuous variables were done using student's $t$-test with Levine's homogeneity of variance or Mann-Whitney's U test as appropriate. Survival was estimated by Kaplan-Meier analysis with the Log Rank test for significance. Comparison of post-operative echocardiograms to baseline values was performed using the paired student's $t$-test. Cox proportional hazards modeling was used to evaluate predictors of survival. SPSS 13.0 statistical software (IBM, Chicago, IL, USA) was used for data analysis and $\mathrm{P} \leq 0.05$ was the criterion of significance.

\section{Results}

A total of 57 patients underwent ITV surgery during the study period. Mean age was $54.4 \pm 14.9$ years (range $23-83$ years). Congestive heart failure was present in $38.6 \%(22 / 57)$, and $43.9 \%(25 / 57)$ were NYHA in class III/IV. The estimated EuroSCORE was $4.7 \pm 6.0$. One patient required an emergent operation. Table 1 shows preoperative baseline characteristics for the entire cohort and for those who underwent repair versus replacement. Eighteen patients underwent $\mathrm{TVr}$ and the TV was replaced in 39 patients $(68 \%)$. There was no statistical difference in baseline characteristics between the TVR and TVr groups. The majority of patients, 54\% (31/57) had history of at least one prior cardiac operation with $61 \%$ [11] of the repair group and $51 \%$ [20] of the replacement group $(\mathrm{P}=0.78)$. Of these reoperative cases, $29 \%(9 / 31)$ had prior TV surgeries (5 prior TVR, 4 TVr) (Table 1).

\section{Etiologies}

The majority of our patients $(79 \%, 45 / 57)$ had severe TR of whom $52 \%$ had primary TV regurgitation. The primary indications for operative intervention were isolated $\mathrm{TV}$ endocarditis $25 \%(15 / 57)$, persistent TV insufficiency after left sided surgery $21 \%(12 / 57)$, traumatic biopsies and iatrogenic injury from pacing leads 19\% (11/57), orthotopic 


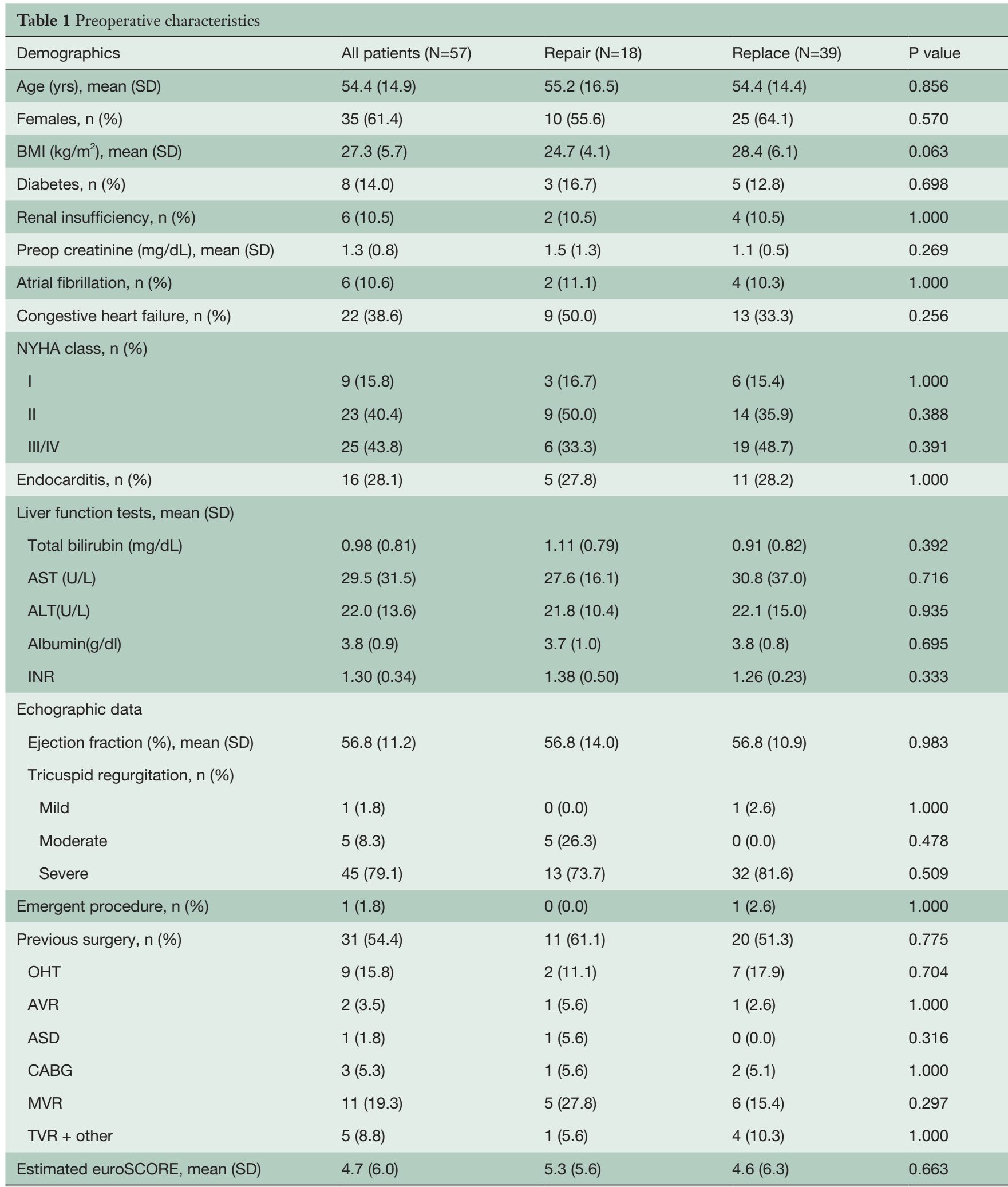

NYHA, New York Heart Association, OHT, orthotopic heart transplant; AVR, aortic valve replacement; ASD, atrial septal defects; CABG, coronary artery bypass graft; MVR, mitral valve replacement; TVR, tricuspid valve replacement; SD, standard deviation. 


\begin{tabular}{ll} 
Table 2 Etiologies of TV regurgitation & \\
\hline Etiologies of TV regurgitation & $\mathrm{N}=57$ \\
\hline Primary & 30 \\
Endocarditis & 15 \\
Pacing wires/biopsies & 6 \\
Blunt chest trauma & 5 \\
Carcinoid disease & 3 \\
Ebstein anomaly & 1 \\
\hline Secondary & 27 \\
Left heart disease (with/out prior surgery) & 13 \\
RV failure (infarction, pericarditis, ASD) & 5 \\
Orthotopic heart transplant & 9 \\
\hline TV, tricuspid valve; RV, right ventricle; ASD, atrial septal defect.
\end{tabular}

heart transplant (OHT) in 16\% (9/57), carcinoid syndrome $5 \%(3 / 57)$, congenital malformations $5 \%(2 / 57)$, and idiopathic TV insufficiency in $9 \%(5 / 57)$. The remaining $48 \%$ had secondary or functional TR as a result of left sided heart failure and pulmonary hypertension (Table 2).

\section{Operative characteristics}

Operative data for the two groups are summarized in Table 3. Cardiopulmonary bypass time and aortic cross-clamp time were similar for both TVr and TVR groups. Of the 39 patients undergoing replacement, 34 (86.1\%) received bioprosthetic valves and $5(13.9 \%)$ received mechanical valves. In the repair group, 61\% (11/18) had ring annuloplasty alone, $17 \%(3 / 18)$ patients had bicuspidization of the TV and $17 \%(3 / 18)$ had De Vega type repair. One

\begin{tabular}{|c|c|c|c|c|}
\hline Variables & All patients $(\mathrm{N}=57)$ & Repair (N=18) & Replace (N=39) & $P \leq A$ vs. $B P$ values \\
\hline \multicolumn{5}{|l|}{ Operative data } \\
\hline Perfusion time (min), median (IQR) & $125(82,167)$ & $125(55,161)$ & $126(89,183)$ & 0.559 \\
\hline Cross-clamp time (min), median (IQR) & $60(45,82)$ & $56(33,79)$ & $64(47,83)$ & 0.500 \\
\hline Bioprosthetic & & & $34(86.1)$ & \\
\hline Mechanical & & & $5(13.9)$ & \\
\hline \multicolumn{5}{|l|}{ Repair, n (\%) } \\
\hline Ring annuloplasty & & $11(61.0)$ & & \\
\hline Vegetectomy & & $1(5.0)$ & & \\
\hline \multicolumn{5}{|l|}{ Postoperative complications } \\
\hline Reoperation for bleed, n (\%) & $1(1.8)$ & $1(5.6)$ & $0(0.0)$ & 0.316 \\
\hline Stroke, n (\%) & $0(0.0)$ & $0(0.0)$ & $0(0.0)$ & - \\
\hline Deep Sternal Wound Infection, n (\%) & $1(1.8)$ & $1(5.6)$ & $0(0.0)$ & 0.316 \\
\hline Sepsis, n (\%) & $2(3.5)$ & $0(0.0)$ & $2(5.1)$ & 1.000 \\
\hline New onset renal insufficiency, $\mathrm{n}(\%)$ & $8(14.0)$ & $2(11.1)$ & $6(15.4)$ & 1.000 \\
\hline ICU stay (hrs), median (IQR) & $53(40,117)$ & $50(45,103)$ & $69(29,127)$ & 0.885 \\
\hline
\end{tabular}




\begin{tabular}{ll} 
Table 4 Surgical approach & $\mathrm{n}$ \\
\hline Approach & $21 / 26$ \\
\hline Surgical approach [no prior surgery $(\mathrm{N}=25) /$ reoperations $(\mathrm{N}=32)]$ \\
Sternotomy & $2 / 1$ \\
Mini-sternotomy & $2 / 4$ \\
Mini-thoracotomy & $0 / 1$ \\
Transcatheter & \\
Cannulation technique (N=57) & 26 \\
Ao/SVC-IVC & 12 \\
FA/SVC-FV & 10 \\
Ao/SVC-FV & 3 \\
Right axillary A/SVC-FV & 2 \\
Right axillary A/SVC-IVC & 2 \\
FA/FV-IJ & 2 \\
\hline FA/FV (advanced to SVC) & \\
\hline Ao, aorta; SVC, superior vena cava; IVC, in &
\end{tabular}

Ao, aorta; SVC, superior vena cava; IVC, inferior vena cava; FV, femoral vein; FA, femoral artery; IJ, internal jugular vein. patient with a history of intravenous drug use presented with active endocarditis had vegetectomy on the septal leaflet and chordae of the anterior leaflet, leaving the valve with very mild regurgitation.

\section{Surgical approach}

Surgical approaches and cannulation techniques are outlined in Table 4. Midline sternotomy was the most common approach in patients with and without prior surgery, $81.3 \%$ (26/32) and 84\% (21/25), respectively. Access to the right atrium was obtained through mini right thoracotomy and lower mini sternotomy in two patients undergoing first time heart surgery versus four mini right thoracotomies, one lower mini sternotomy, and one transcatheter approach in a reoperative patient. Standard aortic and bicaval cannulation was used in $43 \%(25 / 57)$. It was also favored in first time operations $68 \%(17 / 25)$. For patients deemed to be high-risk reoperative candidates, cannulation through femoral vessels was used. A total of $50.9 \%(29 / 57)$ of the operations were performed on the beating heart without aortic cross clamp.
A

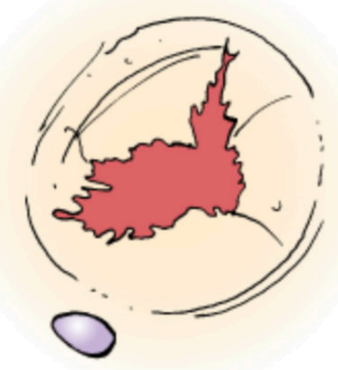

B

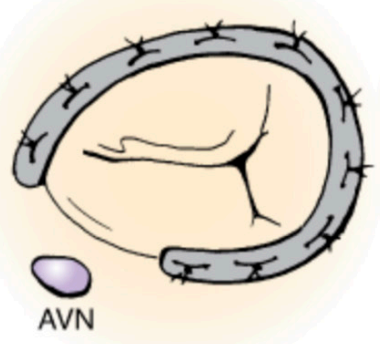

C

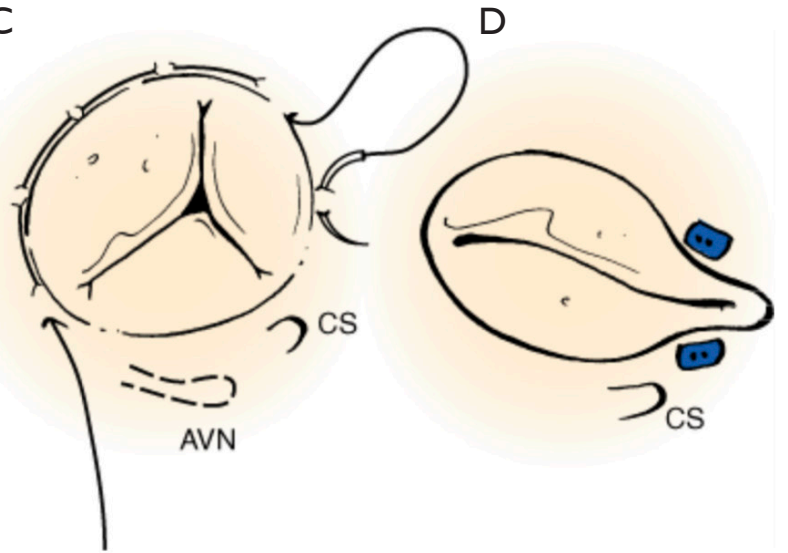

Figure 1 Predominant surgical repair techniques for functional tricuspid regurgitation (TR) in the presence of a dilated annulus. (A) Dilated tricuspid annulus with abnormal circular shape, failure of leaflet coaptation, and resultant TR; (B) rigid or flexible annuloplasty bands are used to restore a more normal annular size and shape (ovoid), thereby reducing or eliminating TR. The open rings spares the atrioventricular node (AVN), reducing the incidence of heart block; (C) DeVega suture annuloplasty partially plicate the annulus reducing annular circumference and diameter; (D) suture bicuspidalization is performed by placement of a mattress suture from the anteroposterior to the posteroseptal commissure along the posterior annulus. CS, coronary sinus. From: Shemin RJ. Chapter 46. Tricuspid Valve Disease. In: Cohn LH. editor. Cardiac Surgery in the Adult, 4e. New York, NY: McGraw-Hill, 2012. With permission from McGraw-Hill. 

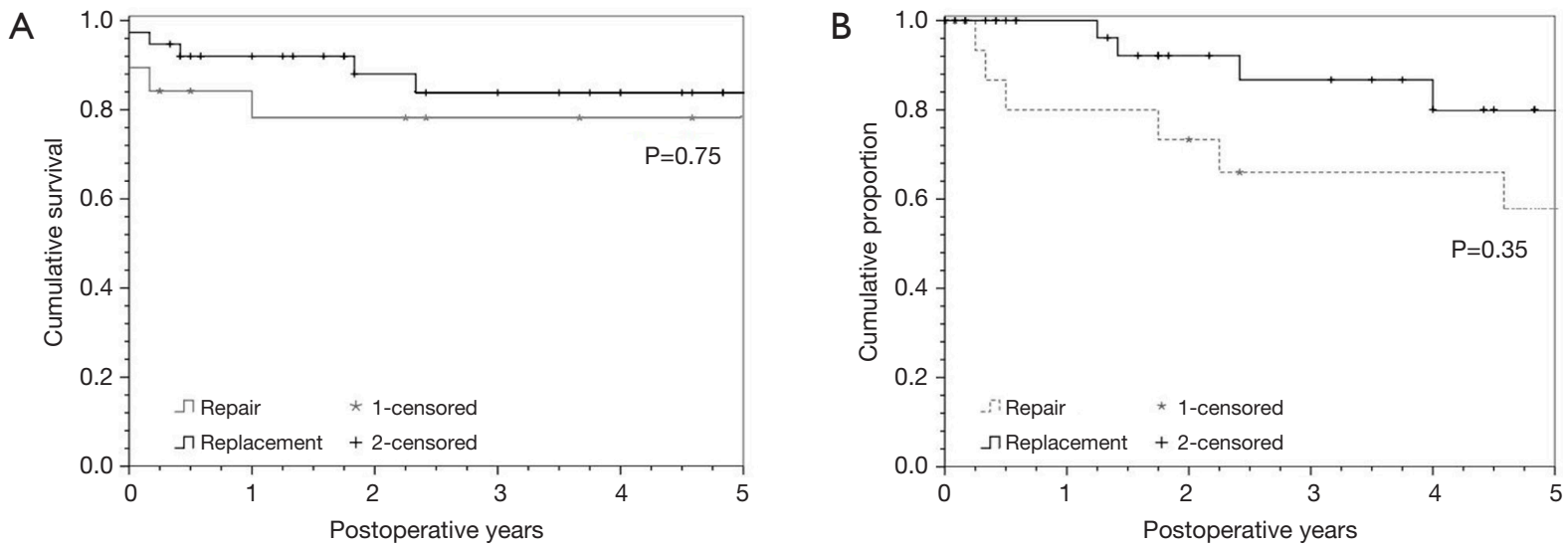

Figure 2 Survival curves for the entire cohort. (A) Showing overall postoperative survival between isolated tricuspid valve repair and replacement; (B) composite proportion of patients without return of moderate/severe TR/RH failure or TV reoperation.

Figure 1 illustrates the types of TVr techniques utilized.

\section{Postoperative outcomes}

Overall operative mortality was $8.8 \%(5 / 57)$. Operative mortality was $5.1 \%(n=2)$ and $16.7 \%(n=3)$ in the TVR and $\mathrm{TVr}$ groups, respectively $(\mathrm{P}=0.312)$. There were no strokes. Eight (14\%) patients developed new onset renal failure but only two required permanent dialysis. Median ICU stay was 53 hrs (IQR 40-117 hrs) and median postoperative length of stay (LOS) was nine days (IQR 6-17 days) (Table 3). There were no statistical differences in outcomes between the $\mathrm{TVr}$ and TVR groups. One patient returned to the operating room on post-operative day one for a right hemothorax but no source of bleeding was identified. One patient with a history of chest radiation for Hodgkin's lymphoma undergoing TV bicuspidization developed a deep sternal wound infection (DSWI) and subsequent multisystem organ failure.

Of the 52 surviving patients, echocardiographic data was available for $48(92 \%)$ with a median time to echo of 2.2 years (IQR 0.5-5.1 years). Recurrent moderate or severe TR occurred in $35.7 \%(5 / 14) \mathrm{TVr}$ patients and $17.6 \%(6 / 34)$ TVR patients $(\mathrm{P}=0.34)$. Among patients with severe TR at baseline, more patients undergoing repair had severe TR at follow-up, compared to those undergoing replacement (61.5\%, 8/13 vs. $14.8 \%, 4 / 27$ respectively, $\mathrm{P}<0.008)$.

\section{Postoperative survival}

Median actuarial survival for the entire cohort was 9.5 years (IQR 8-10.9 yrs). TVr survival at one and three years was
$83 \%$ and $77 \%$ respectively and $91 \%$ and $88 \%$ in the TVR group (Figure $2 A, \mathrm{P}=0.75$ ). Due to the small numbers of patients that required reoperations or had recurrent $\mathrm{TV}$ insufficiency, a composite event-free outcome was defined. Event-free survival was defined as survival without recurrent TV regurgitation, reoperation, or RH failure Figure $2 B$. While the replacement group appeared to have better event-free outcomes, this difference was not statistically significant $(\mathrm{P}=0.35)$.

Cox proportional hazards analysis was performed to identify predictors of poor survival for isolated TV surgery. Predictors of worse postoperative survival included NYHA class IV (HR 16.393; 95\% CI, 13.593-19.194, P<0.001), prior cardiac surgery $(\mathrm{HR}=9.838 ; 95 \% \mathrm{CI}, 7.790-11.886$, $\mathrm{P}=0.029$ ), and total bilirubin (HR $=2.134 ; 95 \% \mathrm{CI}, 1.681-$ 2.587, $\mathrm{P}<0.001)$. Age, ascites, preoperative diuretic use, renal insufficiency, and RV dysfunction were found to be non-contributory, as was valve repair versus replacement $(\mathrm{P}=0.75)$.

\section{Discussion}

Patients undergoing ITV operations are at relatively higher operative risk because of high rates of previous cardiac operations, NYHA class $\geq$ III, and the etiologies requiring TV interventions, including prior left sided valve surgery and iatrogenic causes such as pacemaker lead damage or endomyocardial biopsies.

Eighty-six percent of TV operations are performed in conjunction with another major procedure, usually left sided valve operations, highlighting the relative rarity 
of isolated TV surgery (1). Indications to intervene for isolated TV disease remain poorly defined, and reported outcomes are limited. According to the 2014 ACC/AHA recommendations, indications for isolated $\mathrm{TV}$ procedures includes patients with symptomatic, severe TV regurgitation or asymptomatic with progressive RV annular dilation (>40 mm) and/or systolic dysfunction (2). Symptoms generally include dyspnea due to congestive heart failure, tachyarrhythmia intractable to other therapy, progressive cardiomegaly, and other associated cardiac pathology. Timing of surgery for TV regurgitation is dependent on the etiology of TV disease, which can be divided into two main categories of primary and secondary TR, and degree of RV dysfunction. For patients with symptomatic primary TV regurgitation unresponsive to medical therapy, TV surgery is generally recommended before the onset of significant RV dysfunction (2). Due to extent and severity of the underlying pathology, we generally replace the TV in patients with carcinoid and Ebstein's anomaly.

The incidence of tricuspid regurgitation after OHT has been estimated to range from 19 to $84 \%$. Nine of our patients had previous OHT. Two underwent TV repair, while seven underwent TV replacement (Table 1). The exact etiology of TV regurgitation after OHT is unknown. Anastomotic technique, donor/recipient size characteristics, number of cellular rejections, and iatrogenic injury during endomyocardial biopsies have all been implicated (9). TR at the time of OHT has been shown to predict poor late survival, though it is unclear if TV interventions at the time of OHT will improve long-term outcomes (10). In our series, there was a $33 \%(3 / 9)$ mortality in the OHT group at follow up.

Trauma is a rare but well described etiology of TV incompetence $(11,12)$. TV pathology after blunt trauma is less common than aortic valve injury (12) and medical management is possible if TR is well tolerated. Indeed, post-traumatic median time to operation was 14.5 years (range, 5 months -36 years) for four patients.

Carcinoid valvular disease was present in $5 \%(3 / 57)$ of our patients. One of these patients died, reflecting the known high mortality associated with this disease process. Carcinoid disease of the TV occurs in about $40-50 \%$ of patients with carcinoid tumors $(13,14)$ and large reviews have shown risk reduction with tricuspid valve replacement (TVR) (15). Each of our patients with carcinoid valvular disease underwent valve replacement. Death occurred in an extremely high risk patient with metastatic carcinoid syndrome with liver involvement and ascites, who had a prolonged post-operative course but died from complications related to cancer four months postoperatively.

\section{Comparison of outcomes}

At follow up, five patients in the repair group had failures of the repair with recurrent moderate/severe TR. Of these five patients, two had bicuspidization and three had ring annuloplasty. Published results are mixed regarding the superiority of different repair techniques for the TV, but a review of the literature suggest that ring annuloplasty and De Vega annuloplasty are superior to bicuspidization (16-21). Residual RV dysfunction was also more common in the repair group, $14.3 \%$ vs. $5.9 \%$ in the replacement group $(\mathrm{P}=0.57)$. These differences in residual $\mathrm{TR}$ and $\mathrm{RV}$ dysfunction did not translate into a mortality difference between the two groups, likely due to small numbers. Also, patients who underwent TV repair had a higher incidence of postoperative residual TV regurgitation compared to the replacement group. While our $17.6 \%$ rate of residual $\mathrm{TV}$ regurgitation in the replacement group is relatively high, it is within range of the few studies in the literature that report this information. Buzzatti et al. and Pfannmuller et al. reported residual TV regurgitation in $13.9 \%$ and $17 \%$ respectively after TV replacement $(3,6)$. The reasons for this are unclear and could be due to failure or improper remodeling of the RV geometry after TV replacement. Right ventricular geometry remodeling and function has been shown to improve after TV surgery (22).

Others have reported on the results of isolated TV operations infrequently. Oh et al. (23) in a series of 72 isolated TV operations spanning from 1965-2011 reported an overall early mortality of $12.5 \%$; with a mortality rate of $7.9 \%$ for repair vs. $17.6 \%$ for replacement. While Raikhelkar et al. (24) in a series of 56 patients reported an overall in-hospital mortality rate of $14.2 \%$ with $13.8 \%$ vs. $14.8 \%$ for repair and replacement respectively. In our series, the overall early mortality was $8.8 \%$ with $16.7 \%$ and $5.1 \%$ in the repair and replacement groups respectively. Although our overall mortality for isolated TV is lower, like these two prior studies, there was no statistically significant difference when TV repair was compared to replacement. This is likely the result of small numbers, and a meta-analysis of all these studies might show superiority of either repair or replacement.

In the review by Kilic et al. (1), operative mortality for TV surgery had declined from $10.6 \%$ in 2000 to $8.2 \%$ in 
2010 in both unadjusted and risk adjusted analyses. This large study, which included concomitant procedures, showed this trend toward decreased mortality despite increasing patient co-morbidities (1). Our series spans a decade of experience and represents evolving approaches and techniques. We believe our relative low operative mortality of $8.8 \%(5 / 57)$ is the result of careful patient preparation including pre-operative medical optimization.

\section{Study limitations}

The limitations to our study include those inherent to retrospective analyses. As a large referral center our patient population and resources may not apply to all facilities. Also, the heterogeneity of patients who present with TV failure, which is seen in general, make comparisons of TV repair and replacement difficult. However, in our study the preoperative characteristics between the two groups did not significantly differ from each other. Yet our approach of patient selection, use of minimally invasive incisions when appropriate, and conservative cannulation strategies for reoperations are widely applicable in experienced hands and do not require extensive resource utilization. The small size of our series represents the infrequency of this operation but we presented the breadth of etiologies for comparison.

\section{Acknowledgements}

This work was supported by the Departmental Funds.

\section{Footnote}

Conflicts of Interest: The authors have no conflicts of interest to declare.

\section{References}

1. Kilic A, Saha-Chaudhuri P, Rankin JS, et al. Trends and outcomes of tricuspid valve surgery in North America: an analysis of more than 50,000 patients from the Society of Thoracic Surgeons database. Ann Thorac Surg 2013;96:1546-52; discussion 1552.

2. Nishimura RA, Otto CM, Bonow RO, et al. 2014 AHA/ ACC Guideline for the Management of Patients With Valvular Heart Disease: A Report of the American College of Cardiology/American Heart Association Task Force on Practice Guidelines. Circulation 2014;129:e521-e643.

3. Buzzatti N, Iaci G, Taramasso M, et al. Long-term outcomes of tricuspid valve replacement after previous leftside heart surgery. Eur J Cardiothorac Surg 2014;46:7139; discussion 719.

4. Kim YJ, Kwon DA, Kim HK, et al. Determinants of surgical outcome in patients with isolated tricuspid regurgitation. Circulation 2009;120:1672-8.

5. Sung K, Park PW, Park KH, et al. Is tricuspid valve replacement a catastrophic operation? Eur J Cardiothorac Surg 2009;36:825-9.

6. Pfannmuller B, Moz M, Misfeld M, et al. Isolated tricuspid valve surgery in patients with previous cardiac surgery. J Thorac Cardiovasc Surg 2013;146:841-7.

7. Staab ME, Nishimura RA, Dearani JA. Isolated tricuspid valve surgery for severe tricuspid regurgitation following prior left heart valve surgery: analysis of outcome in 34 patients. J Heart Valve Dis 1999;8:567-74.

8. Gosev I, Yammine M, McGurk S, et al. Should Moderateto-Severe Tricuspid Regurgitation be Repaired During Reoperative Left Sided Valve Procedures? Semin Thorac Cardiovasc Surg 2016;28:38-45.

9. Berger Y, Har Zahav Y, Kassif Y, et al. Tricuspid valve regurgitation after orthotopic heart transplantation: prevalence and etiology. J Transplant 2012;2012:120702.

10. Anderson CA, Shernan SK, Leacche M, et al. Severity of intraoperative tricuspid regurgitation predicts poor late survival following cardiac transplantation. Ann Thorac Surg 2004;78:1635-42.

11. Aykut K, Kaya M, Acikel U. Rupture of the tricuspid valve due to smashing the chest into the steering wheel. Ann Thorac Cardiovasc Surg 2013;19:222-4.

12. Mehrotra D, Dalley P, Mahon B. Tricuspid valve avulsion after blunt chest trauma. Tex Heart Inst J 2012;39:668-70.

13. Bernheim AM, Connolly HM, Hobday TJ, et al. Carcinoid heart disease. Prog Cardiovasc Dis 2007;49:439-51.

14. Gustafsson BI, Hauso O, Drozdov I, et al. Carcinoid heart disease. Int J Cardiol 2008;129:318-24.

15. Moller JE, Pellikka PA, Bernheim AM, et al. Prognosis of carcinoid heart disease: analysis of 200 cases over two decades. Circulation 2005;112:3320-7.

16. De Bonis M, Taramasso M, Lapenna E, et al. Management of tricuspid regurgitation. F1000Prime Rep 2014;6:58.

17. Ghanta RK, Chen R, Narayanasamy N, et al. Suture bicuspidization of the tricuspid valve versus ring annuloplasty for repair of functional tricuspid regurgitation: midterm results of 237 consecutive patients. J Thorac Cardiovasc Surg 2007;133:117-26.

18. Khorsandi M, Banerjee A, Singh H, et al. Is a tricuspid annuloplasty ring significantly better than a De Vega's 
annuloplasty stitch when repairing severe tricuspid regurgitation? Interact Cardiovasc Thorac Surg 2012;15:129-35.

19. McCarthy PM, Bhudia SK, Rajeswaran J, et al. Tricuspid valve repair: durability and risk factors for failure. J Thorac Cardiovasc Surg 2004;127:674-85.

20. Rivera R, Duran E, Ajuria M. Carpentier's flexible ring versus De Vega's annuloplasty. A prospective randomized study. J Thorac Cardiovasc Surg 1985;89:196-203.

21. Tang GH, David TE, Singh SK, et al. Tricuspid valve repair with an annuloplasty ring results in improved longterm outcomes. Circulation 2006;114:1577-81.

Cite this article as: Ejiofor JI, Neely RC, Yammine M, McGurk S, Kaneko T, Leacche M, Cohn LH, Shekar PS. Surgical outcomes of isolated tricuspid valve procedures: repair versus replacement. Ann Cardiothorac Surg 2017;6(3):214-222. doi: 10.21037/acs.2017.05.02
22. Mukherjee D, Nader S, Olano A, et al. Improvement in right ventricular systolic function after surgical correction of isolated tricuspid regurgitation. J Am Soc Echocardiogr 2000;13:650-4.

23. Oh TH, Wang TK, Sidhu K, et al. Isolated tricuspid valve surgery at a single centre: the 47-year Auckland experience, 1965-2011. Interact Cardiovasc Thorac Surg 2014;18:27-32.

24. Raikhelkar J, Lin HM, Neckman D, et al. Isolated tricuspid valve surgery: predictors of adverse outcome and survival. Heart Lung Circ 2013;22:211-20. 